it among their twenty-eight publications marked "OFFICIAL;" and the more so inasmuch as its teaching directly tends to overturn the rules which guide seamen in storms and hurricanes, as well as the first principles of atmospheric physics.

\section{GENTILLI'S TACHEOMETER}

$\triangle M O N G$ the instruments exhibited in the South Kensington Loan Collection is one likely to prove of great use in survey-making; it is the invention of $M$. Gentilli, an Austrian engineer, and its main purpose is to accomplish rapid surveys (hence its name) of difficult country. Not only does it survey the ground, but it gives the height and distance of every point surveyed. The instrument itself differs little from an ordinary surveying telescope. A vertical lever, A, is attached to the axis of the telescope by means of a screw, C (in figure); this lever moves the axis through a given angle, which can be exactly adjusted by means of the two stops, $B \mathrm{~B}^{\prime}$, opposite the free end of the lever. The points to be surveyed are marked by a surveying staff, on which are shown in a manner to be visible at a great distance, very minute divisions of a foot. The telescope is pointed to this distant staff of which it measures : $(a)$ the horizontal angle of position, $(b)$ the vertical angle of elevation, $(c)$ the distance of the instrument from the staff. It is the accuracy with which this last

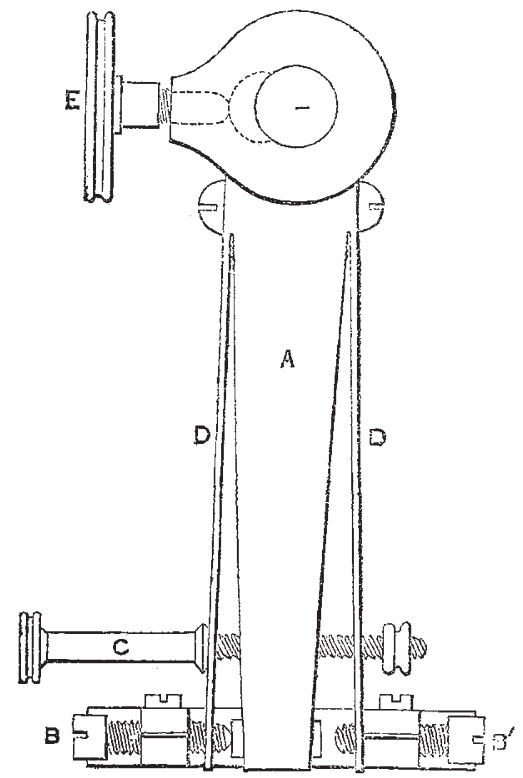

datum can be read that is accomplished by the peculiar mechanism of Gentilli's instrument. As an example : Suppose the staff marked with divisions, to have a scale of 12 feet, on which feet, inches, and eighths of an inch are shown. The telescope is directed to the top of the scale, of which it gives the horizontal and the vertical angle. It is next directed downwards by the screw to a fixed stop, and there it reads on the staff, say to feet 5 inches below the former reading; that distance on the staff is I,000 eighths of an inch, and tells us that the staff is 2,000 yards off. In short, the greater the angle through which the telescope is moved, the greater the distance and vice versâ, Gentilli's telescupe reading the distance and giving it exactly as read, without calculation of any kind. The mechanism is so precise that the telescope can be moved through any given angle and restored to its original position with almost perfect accuracy. Practice has shown that the distances so measured by a small instrument of only 40 magnifying power are correct to within $\frac{1}{000}$ part. The instrument seems likely to be of the greatest use both to ordinary surveyors and to those who have to carry on extensive topographical operations.

\section{THE RADIOMETER IN FRANCE}

A LTHOUGH Mr. Crookes's apparatus was clescribed $A$ in a few French papers at the end of last year, the novelty of the phenomenon has prevented physicists from paying due attention to it till within the last three months. But now the subject has been brought before the Institute and a number of experiments have been made or are being contemplated which are deserving of notice.

The first apparatus in Paris were procured from London, and also from Germany by Geissler; but now they are exported from Paris. There are in Paris not less than three makers-M. Gaeffe, M. Alvergniat, and M. Saleron - who are daily selling the apparatus, so that the instrument will soon become common in all laboratories in spite of the price, which is about 25 francs.

M. Fizeau, the well-known physicist, has stepped forward to defend the theory of air-dilatation. The mos: formidable objection was proposed by M. Ledieu before the Institute. This mathematician ins:sts upon the great fact that in the air at the ordinary pressure the blackened plate is attracted instead of being repelled. He says that there is a decided opposition between these two phenomena, and that at a certain pressure the radiometer cannot move at all. I do not know whether the experiment has been actually tried. The best plan for investigating the question is to construct radiometers in different gases, carbonic acid and hydrogen, which I intend to do. If the rotation is produced merely by dilatation of the residual gas the motion must be quicker in hydrogen and slower in carbonic acid, owing to the difference of conducting power and mobility. But even then it remains to account for the inversion of rotation.

Sometimes the radiometer rotates in an opposite direction without any apparent cause operating upon it. In investigating the question I demonstrated very simply that this is because it emits heat. To obtain inverse rotation it is sufficient to leave it for some length of time exposed to the rays of the sun, or to the radiation of a furnace, and to plunge it in a vessel full of cold water. The effect is immediate, the inversion takes place almost instantly; but the real quantity of heat accumulated in plates being very small indeed, the inverse rotation is accelerated for a few seconds, and diminishes at a very rapid rate. In less than half a minute the radiometer stops. and direct action of the rays causes it to rotate again in the direct way if the vessel is of glass and transparent. The same experiment can be made in the shade, but it requires more caution, as the inverse action is less powerful, and the light can operate with sufficient force to continue the rotation in the normal direction, in spite of reverse force produced by refrigeration. But even in these cases it is possible to perceive a diminution in the rate of rotation. The radiometer falls to a rate which is smaller than the final one, and suffers a visible augmentation after a temporary diminution in the first instance.

M. Alveruniat exhibited, at a recent sitting of the Société de Physique, a double apparatus to demonstrate that the position of the blackened face determines the direction of rotation. The following experiments can be made with a radiometer with both plates blackened, and illustrate the same fact with greater simplicity if the half of the transparent sphere has been previously blackened.

If the blackened hemisphere is perpendicular to the rays, the radiometer will remain motionless; but in an oblique direction it will rotate to the left or to the right, according to the inclination of the incident rays. The least surplus in the quantity of light or heat received by any influenced surface will rotate the apparatus in the direction of repulsion. 